\title{
TORSION TEXTURE MEASUREMENTS WITH HIGH-ENERGY SYNCHROTRON RADIATION ON NiAl
}

\author{
W. SKROTZKI ${ }^{\mathrm{a}, *}$, B. KLÖDEN ${ }^{\mathrm{a}}$, R. TAMM ${ }^{\mathrm{a}}$, C.-G. OERTEL ${ }^{\mathrm{a}}$, \\ U. GARBE ${ }^{\text {b }}$ and E. RYBACKI ${ }^{\mathrm{c}}$ \\ anstitute of Structural Physics, Dresden University of Technology, 01062 Dresden, Germany; \\ ${ }^{\mathrm{b}}$ HASYLAB at DESY, Notkestr. 85, 22603 Hamburg, Germany; \\ ${ }^{\mathrm{c}}$ Geo Research Centre Potsdam, Telegrafenberg, 14473 Potsdam, Germany
}

(Received 13 September 2003)

\begin{abstract}
Diffraction with high-energy synchrotron radiation is a new experimental method to determine textures of materials, which due to the special properties of this radiation, in the future may have advantages in terms of accuracy of local texture measurements in comparison to established methods like Electron back scatter diffraction (EBSD). In the present study NiAl polycrystals with two different initial textures have been deformed in torsion at $727^{\circ} \mathrm{C}$ and $1000^{\circ} \mathrm{C}$ and their texture development has been measured with highenergy synchrotron radiation. Torsion enables the study of texture formation with strain as well as the exploration of large strains without changing the shape of the samples. The pole figures indicate the preferred alignment of $\langle 100\rangle$ with the shear direction and $\{110\}$ with the shear plane. High pressure torsion may also open new possibilities in terms of grain refinement and texture formation and thus ductilization of NiAl.
\end{abstract}

Keywords: Torsion textures; Synchrotron radiation; NiAl polycrystals

\section{INTRODUCTION}

To measure textures in torsion deformed samples, methods with high spatial resolution have to be applied. The method generally used is electron backscatter diffraction (EBSD) allowing orientation measurements of single grains. Although spatial resolution has been steadily improved in the past, limits are given for very small grain size and/or high strains and poor grain statistics. In this article a method is presented which is suitable to go beyond these limitations. High-energy synchrotron radiation, which is available at certain accelerators all over the world, offers the possibility of measuring textures locally with a high resolution because of its large penetration depth. Thus, there is a possibilty of measuring textures strain-resolved in torsion deformed cylinders with a good grain statistics.

In the present study, the analysis of texture development is demonstrated for NiAl. This intermetallic compound has special properties, like high melting point, low

*Corresponding author. 


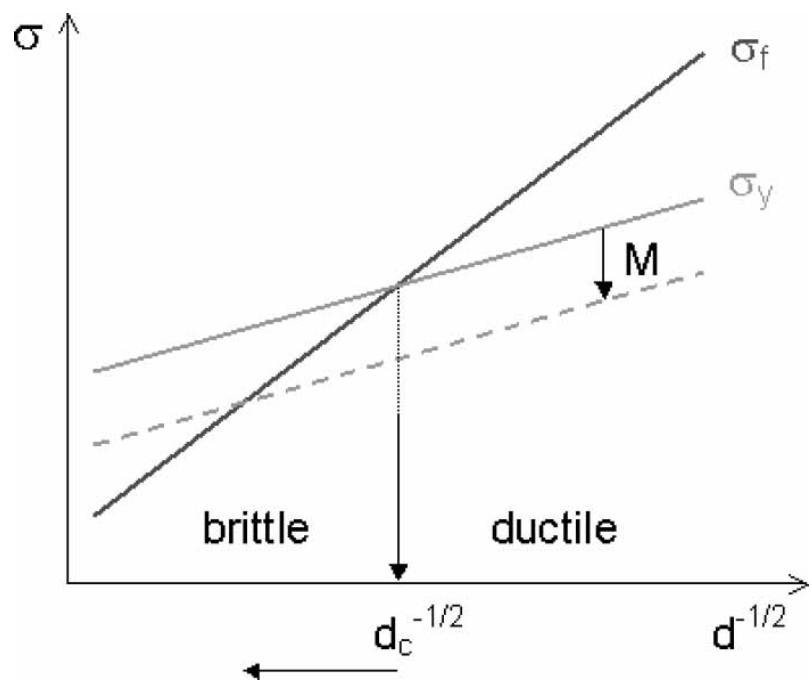

FIGURE 1 Grain size $d$ dependence of the fracture stress $\sigma_{f}$ and yield stress $\sigma_{y}$ defining the brittle-to-ductile transition grain size $d_{c}$. The dashed line shows the effect of texture on $\sigma_{y}$ in the case of a decreasing Taylor factor M. In NiAl, $\sigma_{y}$ is strongly determined by the harder secondary slip systems.

density, good corrosion resistance and moderate creep strength, making this material interesting for high-temperature applications. However, a widespread acceptance as structural material was hindered so far by the inadequate low-temperature toughness and ductility. Attempts to increase the ductility of NiAl by alloying have not been successful (Noebe et al., 1996). Another way to approach this problem is to change the grain structure and texture on purpose. As the yield stress increases much slower with decreasing grain size than the fracture stress, grain refinement should help to reach the ductile field (Fig. 1) (Cottrell, 1958). This field can be extended by changing the texture in such a way that the Taylor factor is reduced. Thus, in order to optimize the desired mechanical properties by thermomechanical treatment, it is of particular interest to understand the mechanisms of grain structure and texture formation during deformation and annealing. To approach this aim, torsion has been used. This deformation mode offers several advantages (Paterson and Olgaard 2000): (i) The local deformation in the specimen is always simple shear, although on the scale of the specimen the shear direction curves around the cylindrical axis. (ii) There is no limit to the amount of twist that can be applied to the specimen. Therefore, the effects of large strains can be explored without significantly changing the geometry of the specimens, thus revealing the influence of strain on the evolution of microstructure and texture. (iii) If strain softening occurs, it may be checked if it is associated with strain localization in shear zones, lattice rotations or dynamic recrystallization.

Emphasis in this article will be put on general problems of torsion texture measurements with synchrotron radiation and problems dealing with data analysis.

\section{EXPERIMENTAL}

The starting material was high-temperature extruded polycrystalline NiAl with two different initial textures. NiAl extruded through a retangular die had an initial preferred 
orientation of $\langle 100\rangle$ parallel to the torsion axis while that extruded through a round die had a $\langle 111\rangle$ fibre parallel to the torsion axis (Fig. 9). The diameter and height of the cylindrical samples were $10 \mathrm{~mm}$. These cylinders were deformed in torsion at $727^{\circ} \mathrm{C}$ and $1000^{\circ} \mathrm{C}$, respectively, in a Paterson-type rock deformation machine (Paterson and Olgaard, 2000) under $400 \mathrm{MPa}$ argon confining pressure. In the following, the low- and high-temperature samples will be denoted LT and HT, respectively, followed by a second attribute indicating the initial texture, e.g. LT 111. The specimens have been deformed between alumina pistons, the whole assembly being enclosed in a thin steel jacket. The applied torsion leads to simple shear in the tangential direction in a plane normal to the torsion axis (Fig. 2). The shear strain and the shear strain rate in the samples increase linearly from zero at the torsion axis to a maximum $\gamma_{\max } \approx 3$ and $\dot{\gamma}_{\max }=2 \times 10^{-4} \mathrm{~s}^{-1}$ at the sample edge. According to the sample dimensions this corresponds to one revolution. To investigate the local textures between the torsion axis and the edge, small pins with a diameter of $1 \mathrm{~mm}$ were prepared in the radial direction for each of the deformed samples (Fig. 2).

Quantitative texture measurements were performed with high-energy $(100 \mathrm{keV})$ synchrotron radiation at the beamline BW5 at DESY-HASYLAB (Hamburg, Germany) (Bouchard et al., 1998; Wcislak et al., 2002). Synchrotron radiation opens a possibility to investigate small sample volumes (local studies). The incident monochromatic beam was defined by a slit system to $1 \mathrm{~mm} \times 2 \mathrm{~mm}$. The small pins were mounted parallel to the rotation axis $\omega$. An image plate detector (MAR 345) was positioned perpendicular to the incoming beam at a distance from the sample of about $1.3 \mathrm{~m}$. Thus, the Debye-Scherrer rings with the indices (100), (110) and (111) could be registered simultaneously (Fig. 3). The pins were rotated continuously around the $\omega$-axis from $-90^{\circ}$ to $90^{\circ}$ and the intensity was summed up for intervals of $\pm 1.5^{\circ}$, resulting in 61 diffraction images. The integral measurement was done in order to account for the extremely low divergence of the synchrotron beam. Thereby each grain is detected in at least one of the diffraction images. The texture after torsion was measured as a function of the shear strain $\gamma$ at five different positions between $\gamma=0$ and $\gamma \approx 3$ along the pin axis shown in Fig. 2. The cylinders radiated had
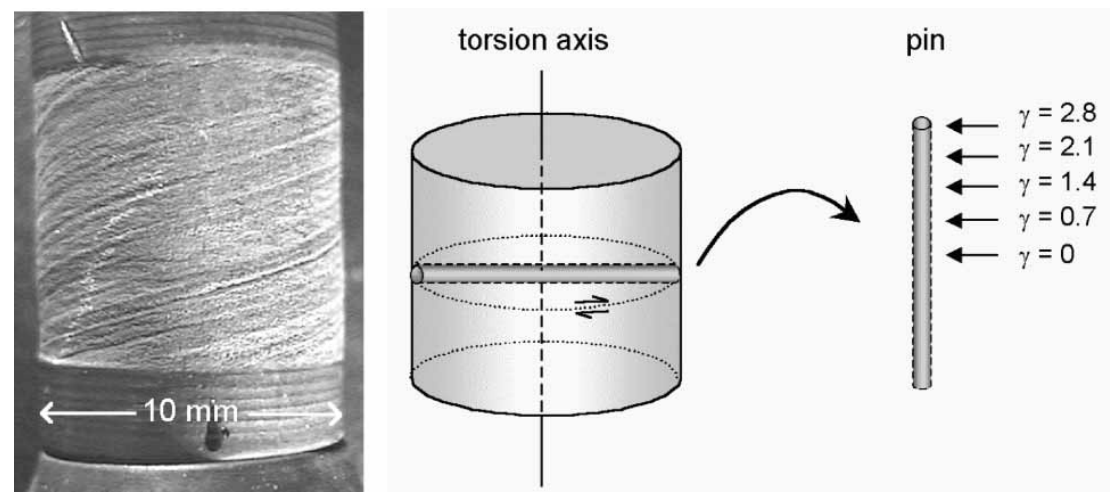

FIGURE $2 \mathrm{NiAl}$ specimen with initial $\langle 111\rangle$ fibre texture deformed in torsion. The rolling traces on the steel jacket were initially parallel to the cylinder axis and serve as passive strain markers to illustrate the simple shear deformation in any tangential plane. A sketch of the position of the pin taken for texture analysis is shown with different shear strains indicated. 


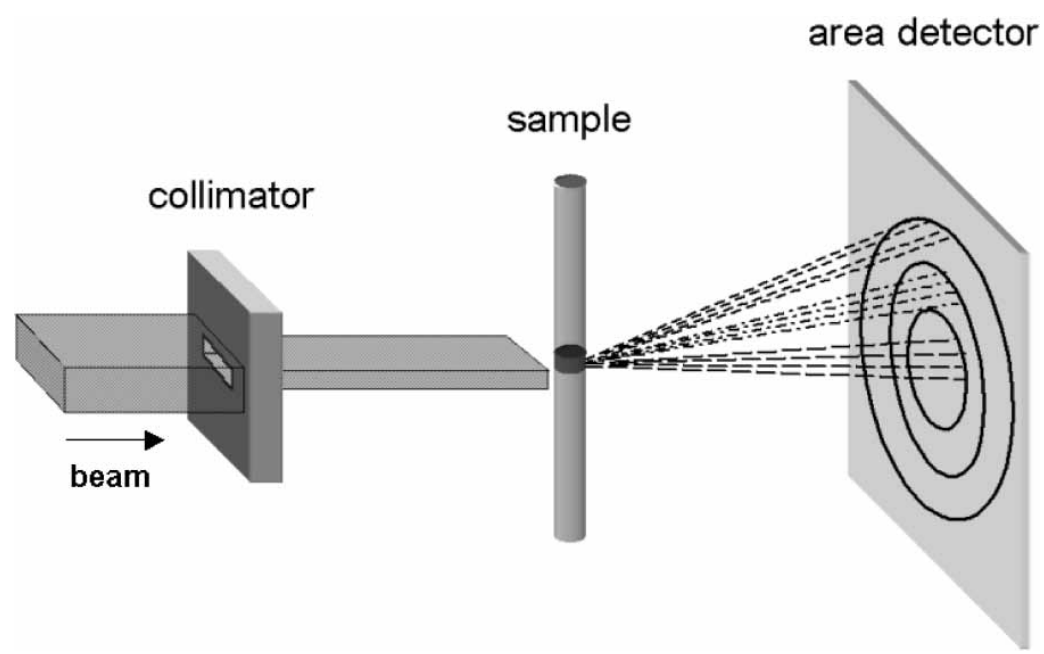

FIGURE 3 Experimental setup for texture measurements with synchrotron radiation (simplified). The beam is confined by the collimator. After going through the sample, the diffracted beam produces a set of Debye-Scherrer rings on an area detector.

a diameter as well as length of $1 \mathrm{~mm}$. Thus, the given shear positions $\gamma$ cover a range of $\gamma \pm 0.3$.

The Debye-Scherrer rings were transformed into the corresponding pole figures as follows. In the first step, the intensity along every ring was read out using a pie-piece like integration box (Fig. 4a), which had an angular width of $5^{\circ}$. For each of the Debye-Scherrer rings, all values of intensity of each pixel in the integration box were summed up. In the second step, the pole figure coordinates for the integration box were calculated (Bunge and Klein, 1996; Wcislak et al., 2002). The resulting pole figure points are on an irregular grid and have to be interpolated onto a regular $5^{\circ} \times 5^{\circ}$ grid (Fig. 4b and c). The interpolation was done in a way that for every point of the $5^{\circ} \times 5^{\circ}$ grid the three nearest neighbours were determined. The distance weighted mean value of the intensities of these three points is assigned to the corresponding grid point. However, by this interpolation procedure a certain percentage of data points is disregarded. An improvement of the interpolation algorithm in order to reduce this problem is in progress. The pole figures shown are recalculated from the orientation distribution function using the program by Dahms and Eschner (1996).

Orientation imaging microscopy (OIM) has been done in the scanning electron microscope (SEM, Zeiss DSM 962) on longitudinal axial sections (Klöden, 2002). The location resolved orientation data were used to calculate the misorientation and grain size distribution. The initial texture of the two samples has been measured by neutron diffraction (Tamm et al., 1998).

\subsection{Problems Related to the Variation of the Shear Direction}

Shear textures are referred to the sample coordinate system defined by the shear plane normal, shear direction and transverse direction. In torsion of bulk cylinders the shear direction circulates around the torsion axis, which coincides with the shear plane 

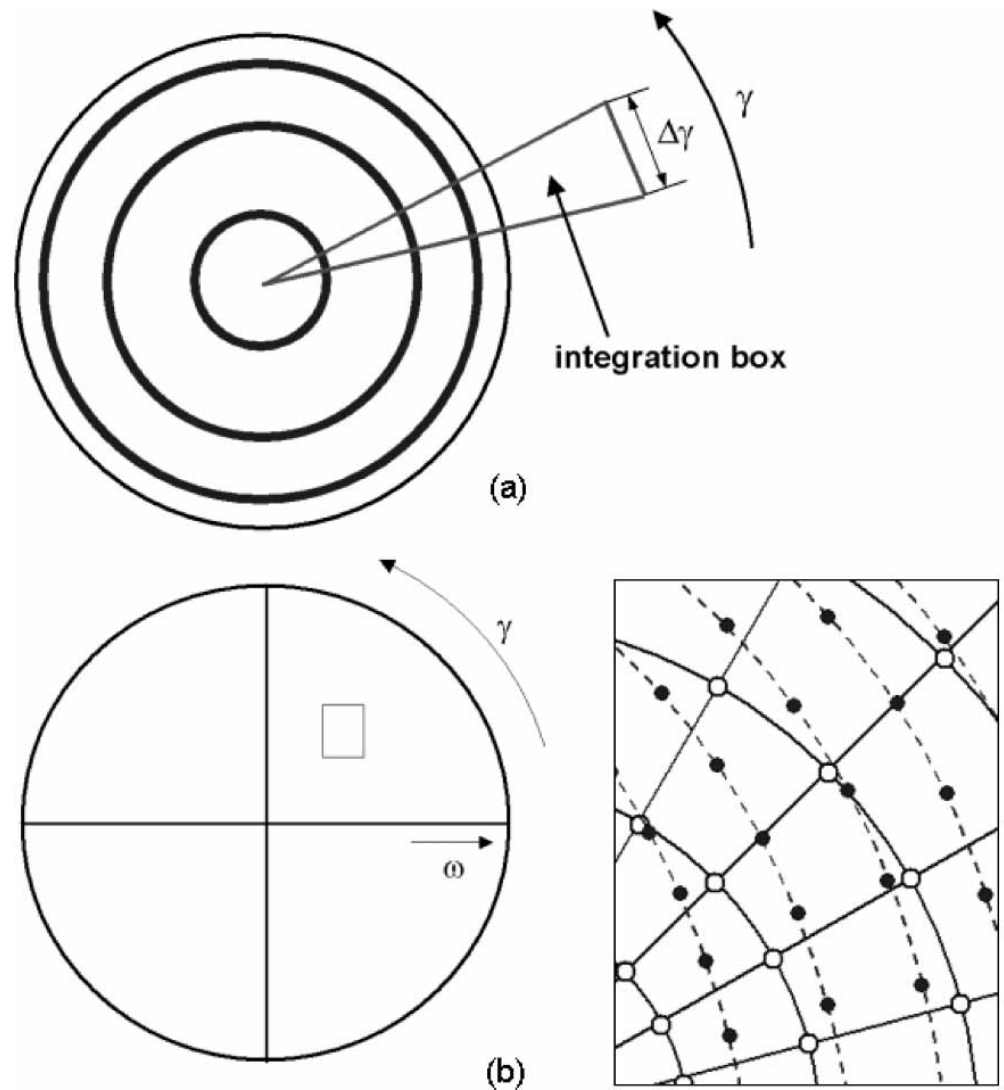

(c)

FIGURE 4 Data analysis of texture measurements with synchrotron radiation. (a) Read out of the DebyeScherrer rings by moving an integration box around the centre of rings in steps $\gamma=5^{\circ}$. (b) The intensity values of each box are converted into the corresponding pole figures and interpolated. The arrows indicate the direction of increasing values of $\gamma$ and $\omega$. (c) Magnification of the rectangular small area indicated in (b). The interpolation and the original grid are shown by open and solid symbols, respectively.

normal (Fig. 5a). Measuring textures in a finite volume element causes a variation of the reference system within this element. This variation increases with increasing size of the volume element and decreasing distance from the torsion axis. Its value is defined by the angle $2 \alpha=2 \times \arctan (d / 2 x)$ ( $d=$ diameter of the pin, $x=$ distance from the torsion axis), which is shown in Fig. 5b. In the pole figures, this variation leads to a broadening of the maxima, especially for those lying in the shear plane.

\section{RESULTS}

The torsion behaviour of the NiAl polycrystals is shown by the shear stress-shear strain curves in Fig. 6. These curves have been calculated from the measured torque and twist data according to the procedure given in Paterson and Olgaard (2000). The curves are characterized by a peak stress followed by moderate strain softening. 

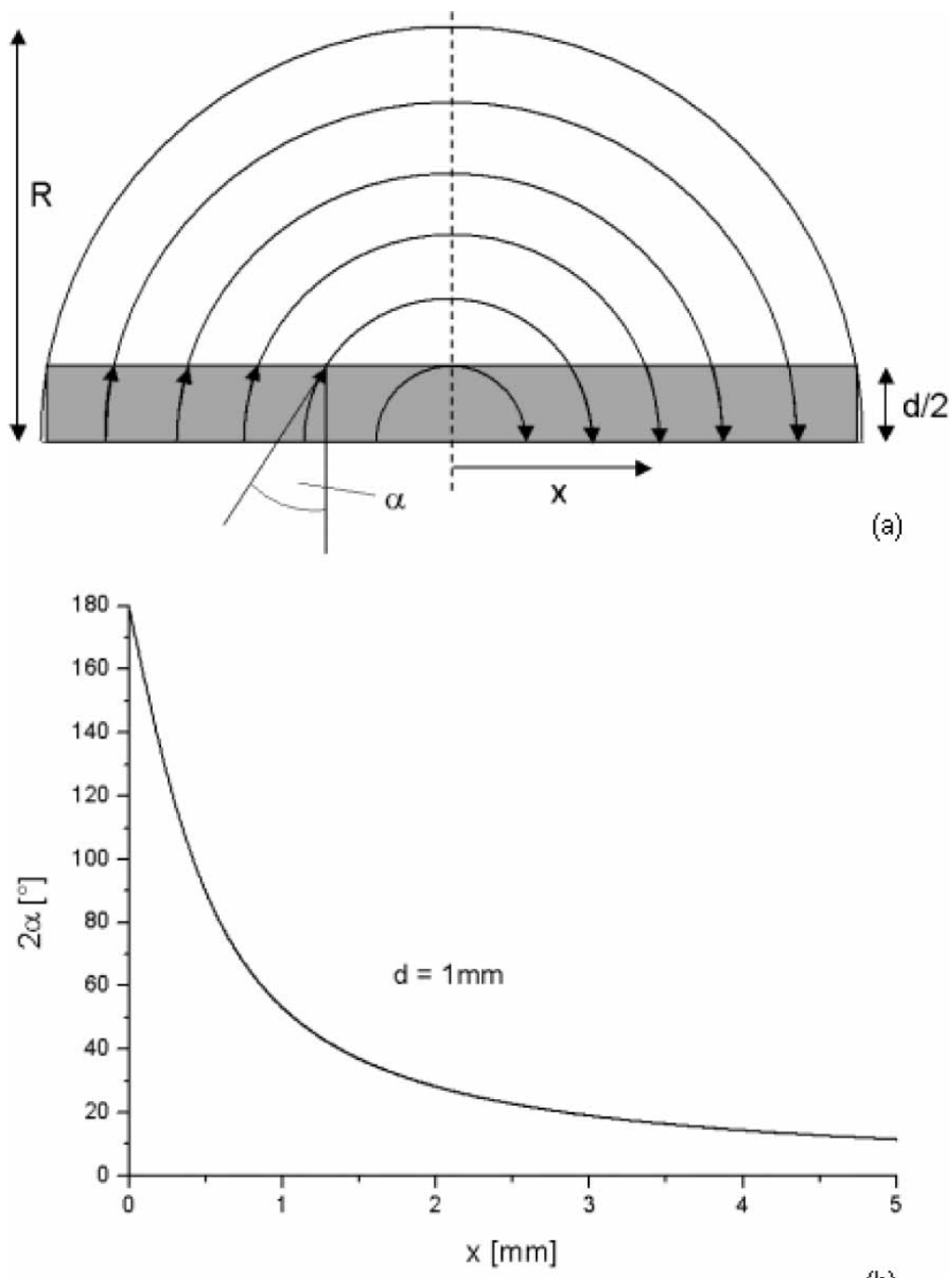

(b)

FIGURE 5 (a) Variation of the shear direction in a pin of radius $d / 2$ (grey area) indicated by arrows (view along the torsion axis). $R$ is the radius of the bulk cylinder, $x$ is the distance from the torsion axis and $\alpha$ is the angle of variation. (b) Relation between $2 \alpha$ and $x$ for $d=1 \mathrm{~mm}$.

As will be shown below, this softening is correlated with textural changes during straining. Due to differences in the initial texture, the $\langle 111\rangle$ samples behave softer than the $\langle 100\rangle$ ones.

The change of microstructure with strain, which has been obtained by OIM, is shown in Figs. 7 and 8. First the microstructure of the LT samples will be described. The initial misorientation distribution $(\gamma=0)$ in the recrystallized state of the extruded sample mainly consists of high angle grain boundaries (HAGB, misorientation angle $\theta \geq 15^{\circ}$ ) (Fig. 7a). With increasing shear strain, low angle grain boundaries (LAGB, $\theta<15^{\circ}$ ) are formed. The fraction of LAGB goes over a maximum, i.e. there is a change in the misorientation distribution towards HAGB (Fig. 7b). The maximum 


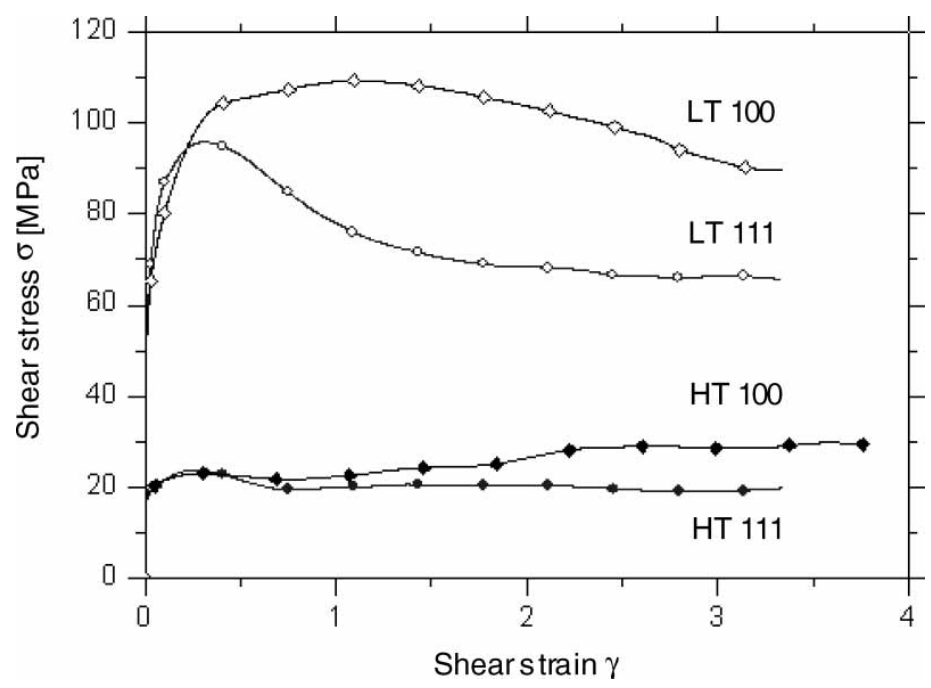

FIGURE 6 Shear stress-shear strain curves of the torsion deformed samples.
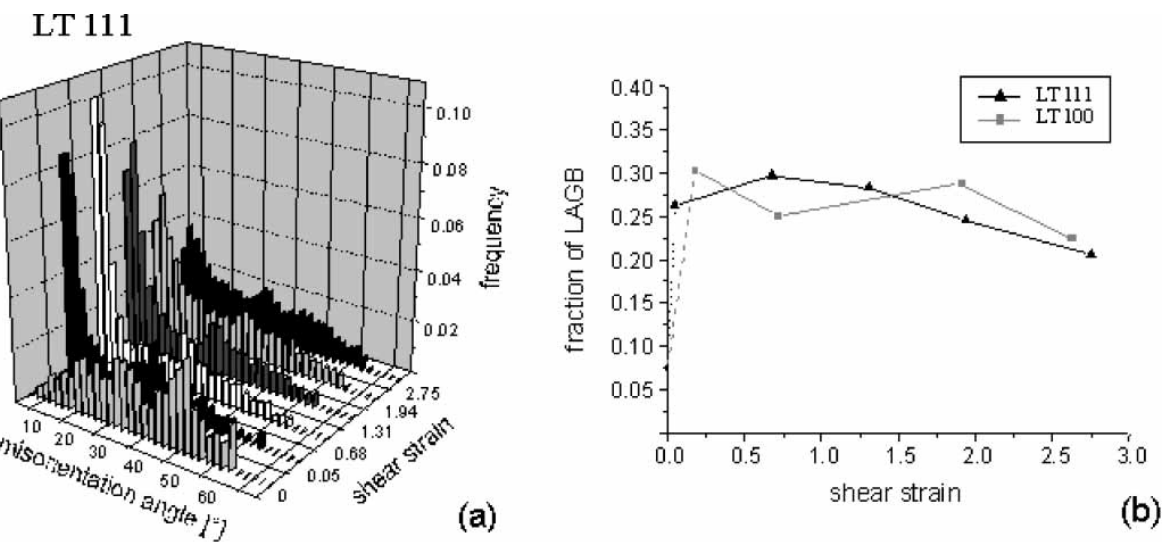

LT 111
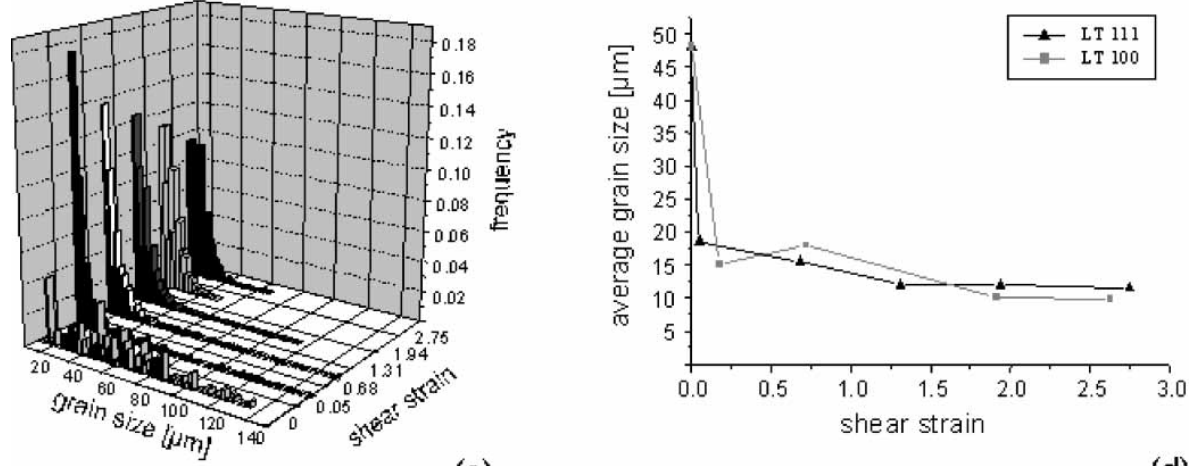

(c)

(d)

FIGURE 7 (a) Shear strain dependence of the misorientation distribution in sample LT 111. (b) Fraction of low angle grain boundaries in samples LT 111 and LT 100. (c) Shear strain dependence of the grain size distribution in sample LT 111. (d) Average grain size in samples LT 111 and LT 100. 


\section{HT 111}

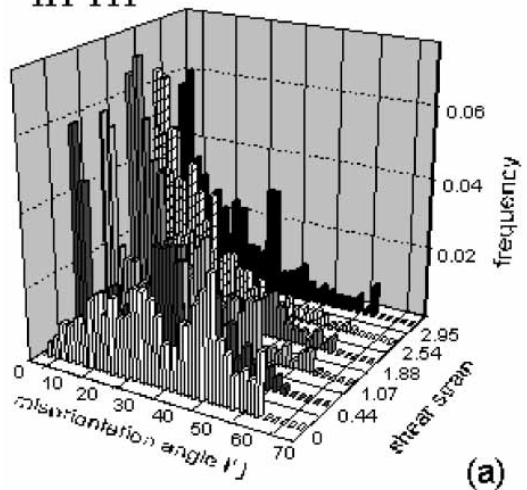

(a)

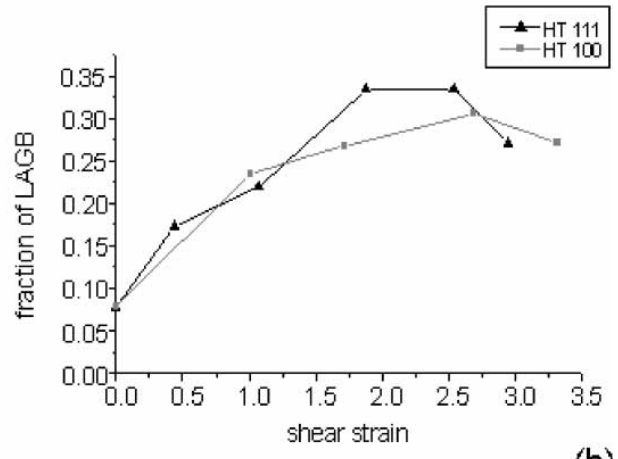

(b)
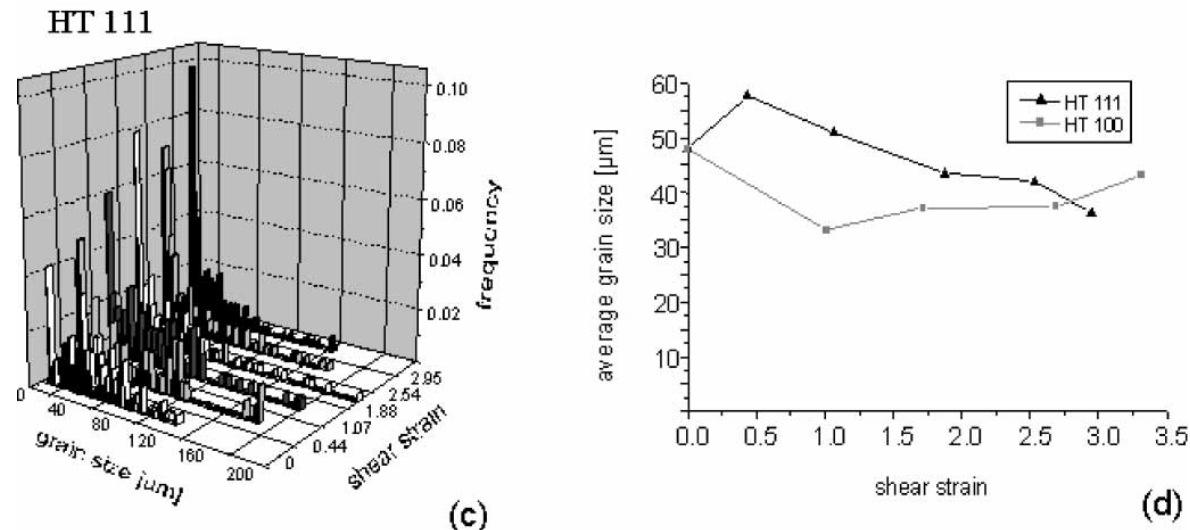

FIGURE 8 (a) Shear strain dependence of the misorientation distribution in sample HT 111. (b) Fraction of low angle grain boundaries in samples HT 111 and HT 100. (c) Shear strain dependence of the grain size distribution in sample HT 111. (d) Average grain size in samples HT 111 and HT 100.

fraction is about $30 \%$. The grain size distribution narrows with inceasing shear strain (Fig. 7c). The average grain size (arithmetical mean) rapidly decreases with shear strain and reaches a steady state value (Fig. 7d). The HT samples show the same tendency with respect to the fraction of LAGB and the value of its maximum (Fig. 8a and $b$ ), but the maximum is reached for higher values of $\gamma$ compared to the LT samples. There are no significant changes in the broadness of the grain size distribution for the HT samples (Fig. 8c). The average grain size remains nearly constant over the whole range of $\gamma$ measured (Fig. 8d).

The texture after torsion was measured as a function of shear strain $\gamma$ at five different positions. The corresponding (100) pole figures are shown for the two different initial textures at four different shear strains (Fig. 9). The given value of $\gamma$ is the one in the middle of the measured sample volume. It varies within this volume by $\Delta \gamma= \pm 0.3$. For all samples, with increasing strain there is an alignment of $\langle 100\rangle$ parallel to the shear direction. The samples LT 111 and HT 111 develop a $\{110\}\langle 100\rangle$ shear texture (notation: \{shear plane\} $\langle$ shear direction〉). This corresponds to an alignment of the primary slip system with the slip plane $\{110\}$ parallel to the shear plane and the 


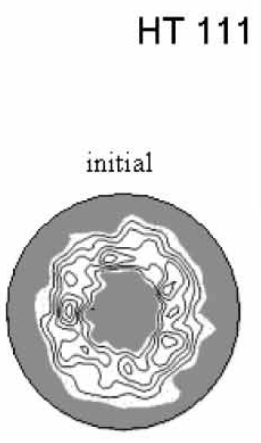

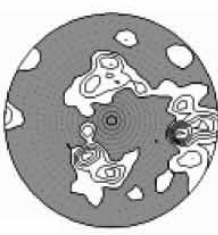

$y=0.3$

$\max =8.8$

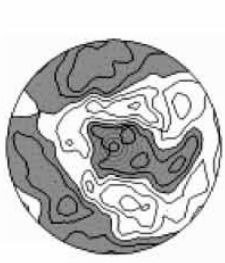

$y=0.1$

$\max =2$

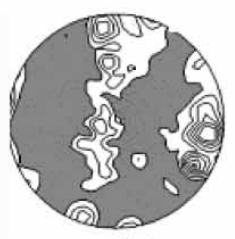

$y=0.9$

$\max =7.6$

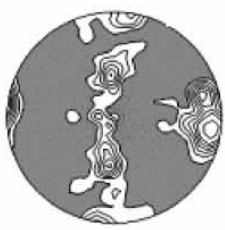

$y=1.7$

$\max =9.8$

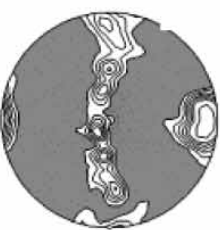

$y=2.4$

$\max =9.6$

LT 111

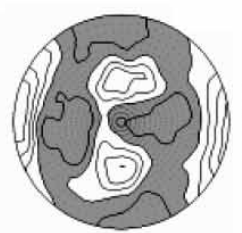

$y=1.4$

$\max =2.8$

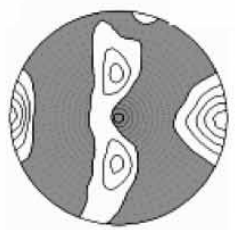

$y=2.1$

$\max =5.7$

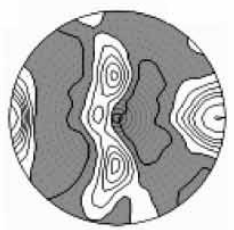

$\gamma=2.8$

$\max =5$

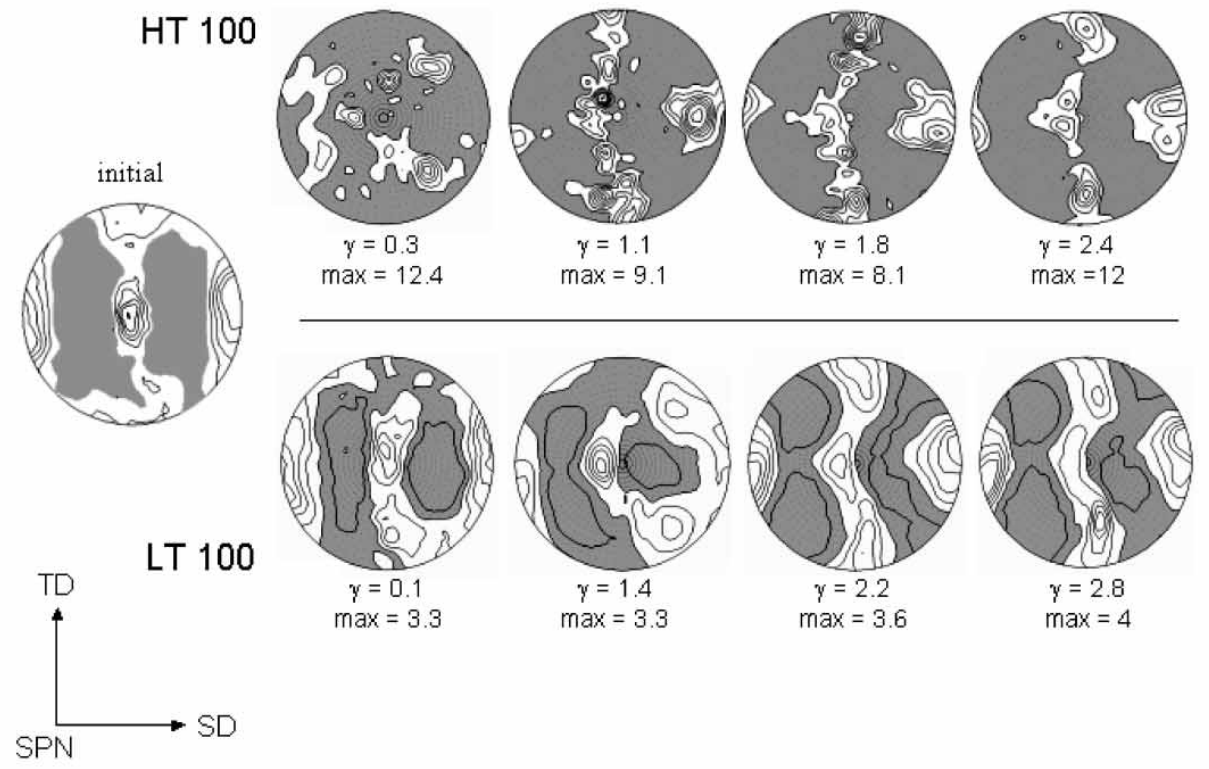

FIGURE 9 (100) Pole figures of the samples for different shear strains. The projection plane is the shear plane $(\mathrm{SD}=$ shear direction, $\mathrm{SPN}=$ shear plane normal, $\mathrm{TD}=$ transverse direction). Maxima are given in m.r.d.

Burgers vector $\langle 100\rangle$ parallel to the shear direction. For the samples LT 100 and HT 100 , there is no similar texture development. While for sample LT 100 the central $\langle 100\rangle$ maximum is decreasing in strength with increasing strain, the two other $\langle 100\rangle$ maxima are beginning to align in a way that $\{110\}$ becomes parallel to the shear plane. This development starts at higher strains for sample HT 100. Except for 
sample HT 100, for low strains the pole figures show similarities with the initial textures.

\section{DISCUSSION}

The microstructure development with shear strain is characterized by subgrain formation in the LT samples. With increasing shear strain more and more dislocations are incorporated into the grain boundaries increasing the misorientation. This process of continuous dynamic recrystallization (Gourdet and Montheillet, 2003) leads to a change from a LAGB to a HAGB structure. As grain boundary migration is negligible the grain size remains about the subgrain size. As this change can be seen for the HT samples as well, but the grain size remains constant, there have to be different reasons for this development. Possibly, an equilibrium between ongoing grain growth and formation of subgrains takes place. This development goes into reverse with increasing strain, but for higher strains than for the LT samples, so there is a change from a LAGB to a HAGB structure as well.

The texture development primarily depends on the initial orientation. As the stressstrain curves indicate a steady state behaviour with increasing strain, a steady state texture is expected too. $\{110\}\langle 100\rangle$ is a likely type of texture, since it represents the alignment of the primary slip system of $\mathrm{NiAl}$ onto the shear plane and shear direction. The tendency of formation of this texture is seen for all samples, but for the samples with $\langle 100\rangle$ as initial orientation higher strains may be necessary in order to reach the steady state texture. The HT samples exhibit an additional axial shortening in the order of 10\% (HT 111) and 16\% (HT 100), which is called Swift effect (Swift, 1947). This effect is superimposed on the shearing and only contributes markedly to the texture development near the torsion axis, where the shear strain is of comparable magnitude. A more detailed study of this effect as well as the influence of larger strains on texture development will be subject of future measurements.

It is a general observation that the steady state subgrain size $d$ is inversely related to the stress $\sigma$, i.e. $d=K / \sigma$ (Skrotzki, 1989). The constant $K$ is independent of temperature and has a value of about $1000 \mathrm{MPa} \mu \mathrm{m}$ for NiAl (Fischer-Bühner, 1998). The measured values of the average grain size are in good agreement with this relationship (Fig. 10). This means that a steady state grain size is already reached for both deformation temperatures. Taking the maximum flow stress at room temperature of about $2000 \mathrm{MPa}$ measured in compression (Skrotzki et al., 2002) results in an extrapolated grain size of about $0.6 \mu \mathrm{m}$. This grain size seems to be the lower bound of grain refinement by deformation unless the mechanism of microstructure development changes with decreasing temperature.

The strain softening observed in torsion may be the result of texture development leading to the preferred alignment of the primary slip system. Grain refinement, unless produced by discontinuous dynamic recrystallization leading to dislocationfree grains, should not affect dislocation creep.

In conclusion, it may be stated that a considerable grain refinement as well as texture formation can be achieved in $\mathrm{NiAl}$ by high-temperature torsion. Further refinement up to two orders of magnitude may be possible by high-pressure torsion (Valiev et al., 2000) at lower temperature. Whether this helps to ductilize NiAl has to be proven. 


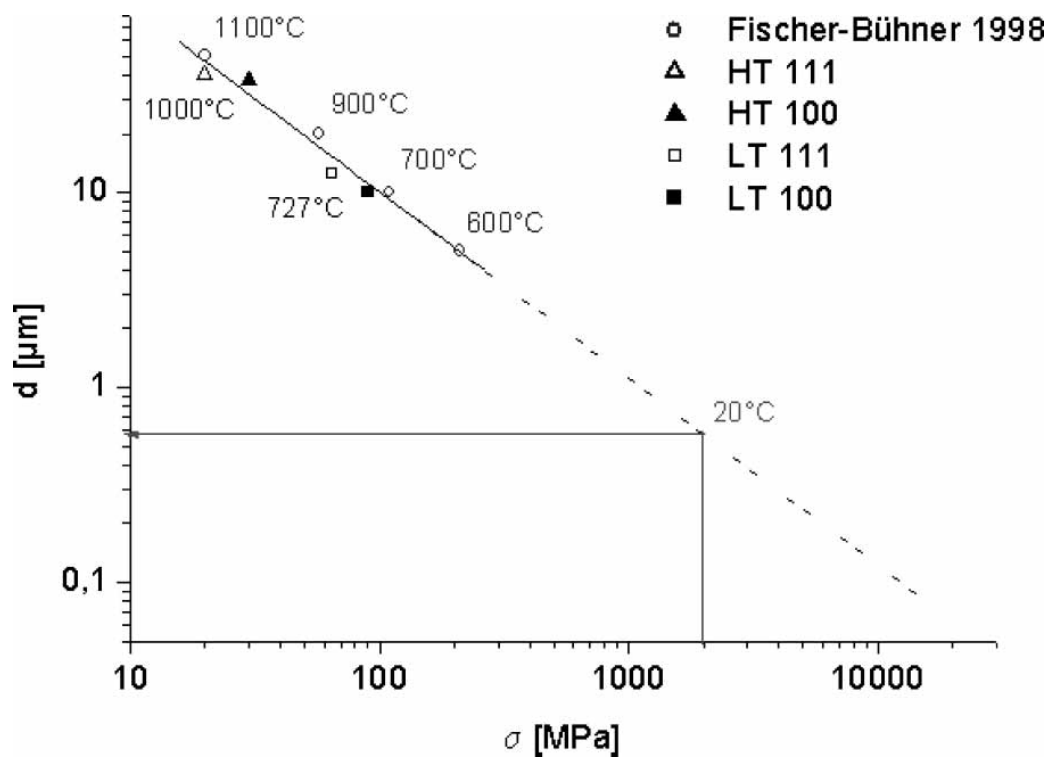

FIGURE 10 Grain size - steady state flow stress relation of NiAl.

\section{Acknowledgements}

Thanks are due to the Deutsche Forschungsgemeinschaft for funding (SK 21/19-1) and DESY-HASYLAB for beamtime and financial support of travel and accommodation.

\section{References}

Bouchard, R., Hupfeld, D., Lippmann, T., Neuefeind, J., Neumann, H.B., Poulsen, H.F., Rütt, U., Schmidt, T., Schneider, J.R., Süssenbach, J. and von Zimmermann, M. (1998). J. Synchrotron Rad., 5, 90-101.

Bunge, H.J. and Klein, H. (1996). Z. Metallkd., 87, 465-475.

Cottrell, A.H. (1958). Trans. AIME, 212, 192-203.

Dahms, M. and Eschner, T. (1996). Quantitative Texturanalyse durch iterative Reihenzerlegung von Beugungs-Polfiguren (software manual).

Fischer-Bühner, J. (1998). Mechanismen der Mikrostruktur- und Texturentwicklung von polykristallinem NiAl. Shaker Verlag, Aachen.

Gourdet, S. and Montheillet, F. (2003). Acta Mater., 51, 2685-2699.

Klöden, B. (2002). Diploma Thesis, TU Dresden.

Noebe, R.D., Bowman, R.R. and Nathal, M.V. (1996). In: Stoloff, N.S and Sikka, V.K., (Eds.), Physical Metallurgy and Processing of Intermetallic Compounds, Chapter 7. Chapman \& Hall, New York, USA.

Paterson, M.S. and Olgaard, D.L. (2000). J. Struct. Geol., 22, 1341.

Skrotzki, W. (1989). The Geological Significance of Microstructural Analyses by Transmission Electron Microscopy. Habilitation Thesis, Göttingen University.

Skrotzki, W., Tamm, R., Oertel, C.-G., Beckers, B., Brokmeier, H.-G. and Rybacki, E. (2002). Mater. Sci. Eng., A329-331, 235-240.

Swift, H.W. (1947). Engineering, 163, 253-257.

Tamm, R., Lemke, M., Oertel, C.-G. and Skrotzki, W. (1998). Mater. Sci. Forum, 273-275, 411-416.

Valiev, R.Z., Islamgaliev, R.K. and Alexandrov, I.V. (2000). Progress Mater. Sci., 45, 103-189.

Wcislak, L., Klein, H., Bunge, H.J., Garbe, U., Tschentscher, T. and Schneider, J.R. (2002). J. Appl. Cryst., 35, 82-95. 